\title{
Forum
}

\section{Le débat sur les OGM : apports et limites de l'approche biologique}

\author{
Marcel Jollivet ${ }^{\mathrm{a}}$, Jean-Claude Mounolou ${ }^{\mathrm{b}}$ \\ a Sociologue, LADYSS/CNRS, Université de Paris 10 - Nanterre, 92001 Nanterre cedex, France \\ b Biologiste, Centre de génétique moléculaire (CGM), CNRS, 91198 Gif-sur-Yvette cedex, France
}

Ce texte à deux voix a pour objectif de poursuivre dans la revue une discussion engagée à plusieurs reprises sur la question controversée des OGM. En débat au sein du comité de rédaction ( $c f$. les deux commentaires), la question des OGM appartient aussi à nos lecteurs. Toute discussion en ce sens sera donc la bienvenue dans nos prochains numéros.

La Rédaction

La question des OGM donne lieu à de multiples discours. Cela tient à la multiplicité des angles sous lesquels elle peut être abordée et à la multiplicité des acteurs sociaux qu'elle concerne (consommateurs, chercheurs, militants d'ONG, industriels, hommes politiques...). Du point de vue scientifique, multiples sont les disciplines, tant du domaine de la biologie que de celui des sciences sociales, dont l'apport est indispensable pour en comprendre les différentes facettes et s'en donner une vue d'ensemble. Mais, dans ce concert, la voix des biologistes occupe une place privilégiée: compte tenu des importants problèmes que posent par ailleurs les OGM sur les plans économique, éthique, environnemental, politique et sociétal, $c^{\prime}$ est sur leurs prises de position que se concentrent attention, attentes et critiques (Joly et al., 2000).

Se cantonnant volontiers dans le rôle que la division $\mathrm{du}$ travail scientifique leur attribue, la plupart d'entre eux s'estiment peu concernés - car pas compétents en la matière - par les questions économiques, juridiques ou politiques que soulève l'utilisation des OGM. Dans une société où la responsabilité des scientifiques est de plus en plus mise en avant, cette attitude peut, à tort ou à raison, passer pour une indifférence regrettable, voire pour un soutien inconditionnel apporté aux OGM. Quant aux controverses auxquelles donne lieu le principe même $\mathrm{d}^{\prime}$ avoir recours à ces derniers, et à la question de leur « acceptabilité sociale » qui est à l'arrière-plan de celles-ci,

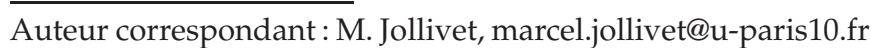

elles ne retiennent au mieux leur attention que s'ils jugent infondés les arguments mis en avant contre les OGM. Ils interviennent alors pour « rétablir les faits ». Ce faisant, ils apparaissent encore, fût-ce - de nouveau - à leur corps défendant, comme des avocats sans réserves en faveur des OGM. Cette posture est d'ailleurs clairement revendiquée par certains.

Une telle vision des choses revient à oublier une donnée essentielle, à savoir que les OGM posent de multiples questions à la science biologique elle-même; nous sommes évidemment là au cœur des différentes spécialités de la discipline, fondamentales et appliquées. C'est oublier également que ces questions - ces incertitudes servent par excellence de fondements aux remises en cause des OGM. Que celles-ci soient le fait de chercheurs scientifiques patentés, biologistes ou non, ou de personnes ne pouvant pas se recommander de ce titre, il s'agit d'une contestation sur une base scientifique. Nous voilà donc en présence de deux discours scientifiques qui s'opposent : celui qui sert de fondement à la défense et à la promotion des OGM, qui émane en particulier de l'« institution » scientifique et qui jouit de son autorité, et celui des opposants, qui apparaît comme un discours militant, fût-il le fait de scientifiques. Une évaluation aussi précise que possible des connaissances et des savoir-faire des biologistes en ce qui concerne les OGM est donc un préalable indispensable à une approche rigoureuse de la question, y compris par les disciplines de sciences sociales. 
On pourrait dire que l'on est là dans le domaine habituel de la controverse, qui est le mode normal de la confrontation scientifique des désaccords. À ceci près que cette confrontation n'a pas lieu. Entre le discours scientifique qui banalise les OGM ou plaide en leur faveur et celui qui s'interroge sur eux ou les diabolise, le ton est plus à la polémique qu'à la controverse. Chacun d'eux mêlant - souvent en vue de s'opposer l'un à l'autre - argumentation scientifique et prise de position sur le fond, leur pertinence et leur légitimité s'en trouvent brouillées. Cette situation et les oppositions sur lesquelles elle débouche sont troublantes si l'on accepte les deux postulats selon lesquels : 1) le fondement de la connaissance scientifique et sa qualité passent par l'acceptation du principe de la confrontation des points de vue et par leur mise en forme claire et réfutable ; 2 ) le monde de la recherche est celui qui est le mieux armé pour procéder à cette mise en forme, laquelle constitue d'ailleurs le cœur et le moteur de son activité. En outre, elles nuisent à la clarification socialement indispensable de la question des OGM.

Il convient donc d'analyser de façon approfondie les décalages entre l'évaluation de l'état des connaissances scientifiques que font les chercheurs favorables aux OGM et celle sur laquelle les opposants fondent leur argumentation et leurs choix.

\section{Quelques précisions préalables}

Une précision méthodologique tout d'abord : nous avons procédé à cette analyse en confrontant plusieurs «corpus » de textes : 1) des textes scientifiques venant de la littérature biologique; 2) des textes d'individus, chercheurs professionnels ou non, ou de collectifs, justifiant d'un point de vue scientifique leur refus des OGM $(\text { Encadré } 1)^{1}$;3) des articles de chercheurs non biologistes portant une interrogation de fond sur les OGM ou sur la biologie.

Par ailleurs, deux ordres de précisions concernant les OGM s'imposent d'entrée pour clarifier la réflexion. Il convient en premier lieu de bien distinguer par des définitions le cadre dans lequel chaque protagoniste se place, sinon des confusions s'installent. Ainsi, d'un point de vue «citoyen », c'est la définition de la loi qui a force de

\footnotetext{
${ }^{1}$ Il convient de préciser qu'aucun souci de « représentativité » n'a présidé à la sélection des textes composant ce second "corpus». Il ne s'agit pas, en effet, de mettre en évidence des différences d'argumentaires liées à des groupes sociaux différents, mais simplement de procéder, sans prétendre à une exhaustivité quelconque, à un inventaire d'arguments suffisamment diversifié pour permettre l'exercice de confrontation entre les corpus. Au demeurant, ce travail de sélection a permis de constater que $l^{\prime}$ on arrivait rapidement à une redondance dans les argumentaires ( $c f$. Encadré 2, liste des questions soulevées).
}

droit. La loi concernée ${ }^{2}$ stipule qu'un «OGM [est] un organisme dont le matériel génétique a été modifié d'une manière qui ne s'effectue pas naturellement par multiplication et/ou recombinaison naturelle». Le biologiste, quant à lui, adopte plus volontiers la définition technique que lui propose 1'Académie des sciences, selon laquelle « un Organisme Génétiquement Modifié (ou transgénique) est créé par l'introduction de manière stable dans ses chromosomes d'une information génétique nouvelle sous forme d'ADN (le transgène) ${ }^{3}$ » (Académie des sciences, 2002). Ici, notons-le, pas de référence à la «nature ». Enfin, pour le consommateur qui est informé de traçabilité et qui lit les étiquettes, un OGM est un produit (alimentaire) dans la composition duquel entrent un ou plusieurs ingrédients issus ou extraits d'un OGM. Cette référence à l'OGM dont provient l'ingrédient en question renvoie de façon équivalente aux deux premières définitions. Mais c'est bien la première qui est dans l'esprit du consommateur.

Tout intervenant dans le débat se réfère nécessairement à l'une ou l'autre de ces définitions. Ce faisant, il se place d'un certain point de vue, affiche une sensibilité propre et marque sa position dans ce débat. Selon le point de vue privilégié, les questions jugées importantes varient. Mais le fait que la définition à laquelle chacun se rattache demeure, comme c'est la plupart du temps le cas, implicite, entraîne une grande confusion. Aussi convient-il de préciser que, compte tenu de l'objectif de ce texte, c'est de la définition de l'Académie des sciences que nous partons.

Les questions soulevées diffèrent aussi, second point, selon que l'on parle de microorganismes, d'animaux ou de plantes OGM. Si tous ces OGM répondent à la définition de l'Académie des sciences et sont issus de travaux de laboratoires, la connaissance qu'on en a et les usages qu'on en fait n'en sont pas aux mêmes stades pour tous. Pour une très grande majorité, ils restent confinés dans ces laboratoires, même si certains d'entre eux, des microorganismes principalement, sont utilisés pour la production de médicaments ou de molécules entrant dans des procédés industriels. Les expérimentations en milieu ouvert ne portent que sur un nombre limité d'OGM végétaux et animaux, et leurs multiplications à grande échelle pour la production (maïs, colza, poissons...), sur des nombres encore plus restreints. De ce fait, les questions scientifiques soulevées changent d'un cas à l'autre.

La distinction entre ces différentes sortes d'OGM s'impose également parce que la façon dont les uns et les autres sont reçus par la société est extrêmement variable (Commissariat général au plan, 2001; Bizet et Pastor, 2003). C'est ainsi que les utilisations industrielles des

\footnotetext{
${ }^{2}$ Il s'agit de la loi no 92.654 du 13 juillet 1992 et de la directive européenne 2001.18.

3 Académie des sciences, 2002, p. 164.
} 


\section{Encadré 1. Corpus}

- «Au nom de la raison », Encyclopédie des nuisances, 12/01/01, 4 p.

- René Riesel, «Aveux complets des véritables mobiles du crime commis au Cirad le 5 juin 1999 », suivi du «Communiqué à

l'issue de l'audience du Tribunal correctionnel de Montpellier consacré au sabotage du riz transgénique du Cirad», 4 p. + 1 p.

- Bertrand Hervieu et Marion Guillou, «Oui aux OGM aux champs », Libération, 23/09/02

- «OGM : Opinion Grossièrement Manipulée », Lettre ouverte à la direction de l'Inra, texte collectif publié par Inf'OGM, avec le soutien de la Fondation Charles Léopold Mayer pour le progrès de l’homme (FPH), octobre 2002, 31 p.

- Jean-Pierre Berlan, « Une cerise sur le gâteau OGM », Le Monde, 05/11/02

- Philippe Desbrosses et al. ${ }^{4}$, «OGM : le quiproquo », Le Monde, 22/11/02

- Hervé Kempf, « Pourquoi faut-il sauver les soldats Bové et Riesel? », Le Monde, 23/11/02

- «OGM et démocratie : chronologie des événements », Le Monde, 23/11/02

- Jean-Yves Nau et Hervé Morin, «Les Académies favorables aux organismes génétiquement modifiés », Le Monde, 14/12/02

- «Une expérience pilote sur les vignes transgéniques : quand le vigneron, le profane et le chercheur délibèrent sur les questions de recherche », Dossier d'information, Paris, Inra, multigr., comprenant : une déclaration de la direction de l'Inra (3 p.), une notice méthodologique ("Quand le vigneron, le profane et le chercheur délibèrent sur les questions de recherche. La méthodologie», 5 p.) et un document intitulé « Rapport final du groupe de travail et réponse de la direction de l'Inra au groupe de travail OGM et vigne et décisions prises sur les programmes de recherche et d'innovation concernant les vignes génétiquement modifiées » $(21+7$ p.)

- «L'expérience pilote OGM-Vigne : un programme de manipulation de l'opinion », réponse au précédent document signée par Nature et Progrès, Confédération paysanne, ATTAC, FNAB, Fédération Rhône-Alpes de protection de la nature - Section Ardèche (FRAPNA-07 ), Groupe international transdisciplinaire (GIET), OGM Danger, février 2003, 10 p.

- Hervé Kempf, « Des agronomes et des généticiens défendent José Bové », Le Monde, 28/06/03

- Jacques Testart, «OGM et entourloupes. De l'hybridation à la biosécurité des plantes modifiées », Le Monde des Livres, 04/07/03, p. IX (compte rendu de l'ouvrage d'Hervé Kempf, La Guerre secrète des OGM, Le Seuil, 2003, 304 p.)

- Jean-Yves Nau, « OGM : pour une surveillance européenne », Le Monde, 05/07/03

- Jean-Yves Nau, « Le pari de l’Europe : suivre la trace des OGM alimentaires », Le Monde, 09/07/03

- Hervé Morin, «La difficile cohabitation des cultures bio, transgéniques et naturelles », Le Monde, 09/07/03 (voir aussi, dans la même page, l'interview de Matthieu Calame, président de l'Institut technique de l'agriculture biologique (ITAB), par Gaëlle Dupont, sur la question de la possibilité ou non de proscrire la dissémination des OGM dans les cultures classiques ou biologiques)

- «OGM. Le règne de l'équivoque», Valeurs mutualistes, Le magazine des adhérents de la MGEN, 226, juillet 2003

- Étienne-Émile Baulieu, «Les sciences à l’heure du soupçon », Le Monde, 22/10/03

- Gaëlle Dupont, «OGM : l'attentisme domine dans le monde agricole», Le Monde, 10/12/03

- Rafaële Rivas, « Les ministres de l’UE devront se prononcer sur le moratoire », Le Monde, 10/12/03

- Gaëlle Dupont, «Les règles de coexistence des cultures restent à établir », Le Monde, 10/12/03

microorganismes OGM se sont développées - et continuent de le faire - sans que cela pose le moindre problème. Leur irruption dans les processus de production est passée inaperçue dans l'opinion. Ils ont même pu jouir d'un préjugé favorable dans la mesure où ils permettaient d'obtenir des produits plus fiables que ceux obtenus par les procédés classiques, comme en témoigne l'exemple des hormones de croissance. C'est à propos des OGM végétaux que les débats ont débouché sur la place publique. Les questions soulevées sont donc souvent spécifiques à ce cas de figure. Mais les débats à leur propos tendent en fait à emprunter un langage général et à servir de tremplin à une remise en question de l'ensemble des OGM. Nous centrerons nos analyses sur les OGM végétaux, et même plus précisément encore sur les plantes OGM destinées à la production agricole, puisque c'est sur eux que porte le corpus étudié, mais nous nous efforcerons de les situer par rapport aux autres et de clarifier à cette occasion les rapports entre unicité et diversité du vivant.

Comme le montre l'Encadré 2, les questions soulevées dans le corpus de textes analysé peuvent être regroupées autour de quatre axes principaux. Cette partition, ainsi que la multiplicité des thèmes abordés dans chacun des axes, illustrent bien la diversité des approches de la question. Une vue d'ensemble a donc son intérêt en ellemême. Ceci dit, nous ne nous intéresserons ici qu'aux controverses à caractère scientifique - et donc d'ordre biologique - portant sur les risques que comportent la

\footnotetext{
${ }^{4}$ Les signataires: Philippe Desbrosses, Yves Dupont, Frédéric Lemarchand, Corinne Lepage, Michel Panoff, Jean-Marie Pelt et Gilles-Éric Séralini sont membres du Comité de recherche et d'information indépendantes sur le génie génétique (CRII-GEN).
} 
Encadré 2. Analyse thématique du corpus

\section{Questions sur les OGM destinés à la production agricole}

1) Quel est l'intérêt réel de ces OGM? Pour les consommateurs (alimentation et santé) ? Pour les producteurs ? Pour l'environnement? Pour le développement?

2) Quels risques pour la santé humaine comportent-ils? Quelle connaissance de ces risques avons-nous?

3) Idem en ce qui concerne les risques pour l'environnement.

4) L'utilisation de plantes OGM réduit-elle réellement le recours aux herbicides?

5) Est-il possible de maintenir étanches une filière OGM et une filière sans OGM?

6) Est-il possible de maintenir une agriculture biologique tout en autorisant les cultures d'OGM?

7) La réglementation de la traçabilité est-elle une solution? Accepter la traçabilité n'est-il pas la porte ouverte à la banalisation des OGM?

8) Les OGM remettent-ils en question le droit des agriculteurs de produire leurs semences (assujettissement des agriculteurs aux firmes)?

\section{Questions portant sur le statut scientifique des OGM végétaux}

9) En quoi consistent les OGM végétaux : entité scientifique ou «chimère »? En quoi cette précision terminologique a-t-elle son importance?

10) En quoi consistent les recherches sur les OGM végétaux : recherche ou ingénierie biologique?

11) Quelles spécialités de la biologie sont concernées? Peut-il y avoir des divergences d'appréciation entre elles?

12) De la sélection végétale aux OGM : continuité ou rupture?

13) Quel est l'intérêt des expériences en plein champ : recherche fondamentale ou appliquée (biotechnologie industrielle)?

\section{Questions sur les orientations de la recherche en biologie}

14) Peut-on dire que la recherche publique sur les OGM est assujettie aux multinationales?

15) Peut-on dire que la recherche publique néglige les alternatives aux OGM?

16) Quelles sont ces alternatives?

\section{Questions sur les implications sociopolitiques des OGM}

17) Peut-on dire que l'introduction des OGM n'a pas respecté les règles de la démocratie (en France) et que la contestation radicale (J. Bové) a joué un rôle positif?

18) Peut-on dire que, de toute façon, les OGM font déjà partie de notre monde et qu'il faut donc s'en accommoder tout en en réglementant l'utilisation?

19) Les OGM sont-ils la porte ouverte à l'eugénisme (cf. le «radicalisme » de R. Riesel)?

mise en culture des OGM végétaux et l'utilisation des produits qui en sont extraits (axe 1). Ce choix se justifie par le fait que ce sont les questions les plus vives et que beaucoup des réponses aux autres découlent de celles qui seront d'abord données à celles-ci.

\section{Des questions connues}

En ce qui concerne les utilisations des OGM (végétaux), l'accord se fait au moins sur la gamme des questions qu'elles soulèvent ${ }^{5}$. En dehors de celles qui tournent autour du principe même d'avoir recours à des plantes OGM, on peut les regrouper en trois blocs : un d'ordre économique (Joly et Hervieu, 2003), un qui concerne la santé humaine (Académie des sciences, 2003) et un qui a trait à la protection de l'environnement (Ministère de l’Écologie et du Développement durable, 2003).

\footnotetext{
${ }^{5}$ Les intérêts que présentent les OGM végétaux sont en fait à examiner sur deux plans, qui doivent être soigneusement distingués : l'un strictement scientifique, l'autre ayant trait à leurs utilisations. Seul le second est examiné ici.
}

Les questions d'ordre économique n'entrent pas dans le champ de notre analyse. Par contre, les attentes et les risques concernant la santé humaine et l'environnement posent des questions qui sont du ressort direct de la biologie. Dans ces deux domaines, ce sont surtout les risques que génèrent les OGM qui donnent lieu à contestation. Les débats qu'ils suscitent sont d'ailleurs connus ; ils tournent autour de questions relatives : 1) à la toxicité éventuelle et au pouvoir allergène des molécules fabriquées par les OGM et leurs combinaisons; 2) au risque d'usages incontrôlés de plantes productrices de molécules médicamenteuses ; 3) aux conséquences dans l'environnement, sur la biodiversité, comme sur les ressources que l'on en tire, de plantes OGM résistantes aux pesticides ou en produisant de nouveaux (Mounolou et Fridlansky, 2002).

Pour les biologistes, aucune de ces questions n'est radicalement nouvelle. Sur toutes, ils disposent de références scientifiques, de connaissances spécifiques (celles de la toxicologie, de l'immunologie, de la physiologie, de la biologie des populations ou de l'écologie) et de moyens d'investigation pour se livrer à un examen au cas 
par cas. Ces questions, formulées en termes de risques, conduisent à qualifier par des probabilités les observations faites comme les projections possibles. Des concepts et des démarches universels, adaptés à de telles problématiques, existent. Ils ont été élaborés dans un effort interdisciplinaire qui bénéficie d'une longue expérience, indépendamment du cas particulier des OGM. Par contre, la mise en œuvre des outils disponibles demande un investissement de recherche spécifique, puis des filières technologiques adaptées, des instances d'évaluation qui définissent des normes et des réglementations, enfin des structures de contrôle. La mise en place de toutes les étapes de ce processus, de la connaissance aux contrôles, exige de la décision, du temps, du travail et de l'argent. Ainsi que le courage de reconnaître qu'il n'y aura, au bout de l'effort, ni réponse définitive ni équilibre confortable, mais une situation nouvelle qui posera ses propres questions et en relancera d'autres ${ }^{6}$.

Le recours aux plantes OGM correspond donc bien à de nouveaux risques sanitaires. Mais ils sont du même ordre de grandeur et de même nature que ceux que toutes les innovations antérieures ont toujours introduits, que ce soit dans le domaine de la santé ou dans d'autres. Sur le plan scientifique, aucune de ces questions ne crée une situation de rupture dans la façon de les prendre en charge. Il s'agit simplement de s'en donner les moyens. En outre, elles ne concernent en rien spécifiquement les OGM végétaux; on pourrait les poser tout aussi bien à propos de cultures de cellules animales ou de microorganismes génétiquement modifiés mis en culture pour produire des médicaments ou intervenir dans de nouveaux procédés industriels. Or, elles ne font débat qu'à propos des premiers. Ignorance de l'utilisation d'OGM par l'industrie? Confiance particulière dans la sûreté des processus industriels qui permettent de mettre à la disposition du public des protéines et non des êtres vivants? Représentation et symbolique de la plante entière et de sa culture? Interférence avec la question des risques écologiques, second volet de la contestation?

\footnotetext{
6 À l'heure actuelle, il n'existe pas de risque toxique avéré concernant les OGM vivants mis dans le commerce, et établir des normes n'a pas de sens. C'est la grande différence avec les pesticides, dont on connaît des effets toxiques pour l'homme et pour lesquels ont été établies des normes : seuil de toxicité, dose journalière admissible, limite maximale de résidu dans un produit commercial, etc. Par conséquent, tout un travail d'analyse de risque et d'élaboration de normes mérite d'être conduit pour les OGM végétaux producteurs de pesticides et les usages des produits qui en sont dérivés. Mais ceci ne peut évidemment se faire qu'à long terme, puisqu'il n'y a pas d'effet avéré à court terme. Ceci n'est qu'un exemple et une approche interdisciplinaire peut, spécifiquement, au cas par cas, proposer des réponses (toujours provisoires) aux inquiétudes que fortifie l'apparition, sur le marché de la drogue, de Cannabis OGM surproducteur de cannabinol...
}

\section{Des risques écologiques maîtrisables}

$C^{\prime}$ est en fait sur ce point que la controverse est la plus intense. On pourrait pourtant facilement dire que, là non plus, la situation n'a rien d'original : les questions sont bien identifiées et partagées (Mounolou et Fridlansky, 2002). Soulignons simplement qu'en l'occurrence, la liste des interrogations va de questions très spécifiques (avantages aux insectes parasites qui acquièrent une résistance aux pesticides avec lesquels les mettent en contact les plantes OGM, dissémination de gènes portés par les plantes OGM à des plantes sauvages de la même espèce ou d'espèces différentes, modifications de la diversité biologique des communautés d'êtres vivants...) à des problèmes plus généraux (invasions biologiques, appauvrissement du patrimoine génétique collectif des espèces, modifications globales de l'environnement par changement des fonctions des espèces qui le peuplent...). Ces risques existent. Et leurs effets peuvent même, sur un plan strictement biologique, être considérés comme créant une situation irréversible. Ils peuvent donc être vus comme irréversiblement «dommageables » si l'on considère que la diversité biologique existante, ainsi que les processus qui, en l'état actuel des choses, la produisent et la font évoluer, présentent des avantages qu'il est indispensable de préserver ( $\mathrm{d}$ 'où la référence à la notion de patrimoine). Précisons toutefois qu'il s'agit là d'un jugement de valeur, c'est-à-dire d'une appréciation qui n'est pas uniquement fondée par la science biologique, mais qui correspond avant tout à une représentation sociale concernant les vitesses et les chemins des évolutions biologiques souhaitables. Du point de vue strictement biologique, l'évaluation que l'on peut faire sur le plan scientifique est semblable à celle qui a été faite sur le point précédent : les biologistes sont outillés pour aborder les questions soulevées.

Ceci vaut pour la question de la résistance acquise par un insecte aux molécules produites par une plante OGM en vue de la protéger de lui : si la démarche reste à faire, les connaissances et les techniques existent. Celles dont nous disposons actuellement ont fait la preuve de leur pertinence, en même temps que de leurs limites (apparitions d'insectes résistants, suivies de leurs disparitions; modifications de l'environnement. . ) à propos d'autres problèmes (par exemple, la lutte contre les moustiques vecteurs du paludisme ou contre les criquets pèlerins ravageurs des cultures). Mais il en va de même en ce qui concerne les incidences biologiques que risquent d'avoir sur l'environnement et l'agriculture l'expérimentation en plein champ des OGM et, plus encore, leur culture à grande échelle (INRA, 2002). On s'arrêtera tout particulièrement ici sur la question qui revient comme un leitmotiv dans ce débat : celle de savoir s'il est possible, « traçabilité » à la clef, de maintenir étanches une filière 
végétale OGM et une sans (question qui se pose aussi pour la préservation d'une "agriculture biologique»).

Cette exigence est d'ordre social et ne doit rien à la biologie. Il n'en revient pas moins à cette dernière de répondre à la question de savoir si - et à quelles conditions - elle peut être honorée. Pour aborder cette question en biologiste, il est indispensable de distinguer deux niveaux, qui sont en même temps deux ordres de grandeur, différents : celui du laboratoire et de la production de semences OGM, celui des cultures sur de grandes surfaces.

\section{Stabiliser une semence : un travail de Sisyphe}

En ce qui concerne le premier niveau, il convient d'être clair : espérer maintenir étanche la séparation entre une filière avec OGM et une sans suppose que l'on soit capable de reconnaître les produits d'une reproduction absolument à l'identique (un clonage parfait) et d'entretenir cette reproduction génération après génération. La biologie affirme que, dans le monde vivant, ceci n'existe pas : le vivant certes se perpétue, mais il se diversifie sans cesse (mutations de l'ADN, recombinaisons et brassages lors de la reproduction sexuée, échanges génétiques « horizontaux » entre espèces). On sait, en biologie, qu'entretenir une variété (de carottes, par exemple) identique à elle-même nécessite un travail de sélection permanent pour choisir parmi les individus d'une génération ceux qui auront conservé, au moins autant que l'on puisse le savoir, le patrimoine génétique des parents, et dont on fera les géniteurs de la génération suivante. En bref, dans cette affaire sont " associés », de façon discrète (puisque le consommateur souvent l'oublie ou ne le sait pas), des biologistes et des généticiens, des producteurs de semences, des agronomes, qui ont établi les conditions de culture et de confinement les plus intéressantes pour les plantes qui porteront les graines dont ont besoin les maraîchers, ainsi que des agents de la puissance publique qui, en réponse aux normes édictées par le législateur, font appliquer les règles $\mathrm{d}$ 'isolement et de contrôle, $\mathrm{d}$ 'attribution de certificats et de labels...

A priori, rien n'indique que les gènes "étrangers " portés par les OGM végétaux aient un comportement différent des gènes de l'hôte. On devrait donc être outillé pour traiter de ces plantes nouvelles. Cela ne dispense pas de les mettre à l'épreuve d'un contrôle scientifique et la référence de base de l'opération est le comportement des gènes de l'hôte, ainsi que la réglementation qui l'aménage. Inutile donc de crier «au loup!» : les biologistes ont les moyens de se doter des connaissances nécessaires et propres à chaque OGM. En France, dans le cadre du moratoire, ce travail a été fait par des scientifiques et des professionnels (INRA, 2002; Semences et biodiversité, 2003). Les résultats, cas par cas, en ont été enregistrés par la puissance publique (Boyaval, 2003). Les réponses, si elles sont prises en considération sans a priori, permettent aux citoyens comme aux politiques de prendre leurs responsabilités... Bien entendu, il s'agit de réponses scientifiques, avec leurs avancées et leurs incertitudes nouvelles (Ternois, 2004) : elles ne sont jamais définitives et ne dispensent pas les décideurs de devoir trancher!

\section{Une question de société soluble dans la biologie}

Traiter des OGM autorisés en grande culture ${ }^{7}$ est une autre question et la vigueur du débat sur ce point peut s'expliquer par l'ampleur des incertitudes et des enjeux que soulève le passage à cette échelle, avec le cortège $\mathrm{d}^{\prime}$ interférences environnementales, économiques et sociales qu'il entraîne $^{8}$. À l'échelle des centaines ou des milliers $\mathrm{d}$ 'hectares, il est en effet démontré que les gènes nouvellement introduits par ces plantes sont distribués à d'autres, en particulier par le pollen, et par conséquent vont avec une fréquence faible se retrouver dans des cultures qui ne les possédaient pas. Cela n'étonne en rien le biologiste qui savait que ceci se passe tout autant avec les gènes d'une variété classique passant chez la voisine... Les agriculteurs étaient depuis des décennies avertis, empiriquement ou techniquement, de ce phénomène qui leur impose périodiquement de renouveler leurs semences.

Pour éviter une banalisation de la pénétration des OGM dans les cultures classiques et agrobiologiques, il convient de compartimenter l'espace et donc de connaître les processus de dispersion des gènes pour fixer les règles de ces partitions. En d'autres termes, les conséquences de la culture d'OGM doivent être étudiées spécifiquement sur la descendance de ces plantes et sur la descendance des plantes voisines non OGM. Là aussi, les bases conceptuelles et les moyens pour le faire existent et la référence scientifique est la connaissance déjà établie de la dynamique de diffusion de gènes déjà connus dans les populations cultivées ou non des plantes de l'espèce. Laisser croire que les biologistes, les agronomes et les professionnels ne savent rien sur le sujet est une imposture.

Mais il est acquis que la réutilisation des semences après plusieurs cycles de culture, OGM ou non, demande une réflexion, un examen et une décision sérieuse. C'est ici qu'interviennent déjà les procédures dites de «traçabilité», des normes officielles qui fixent le taux acceptable

\footnotetext{
${ }^{7}$ Les questions soulevées par la mise en culture concernent aussi, même si les échelles n'ont rien de comparable, les essais en plein champ.

${ }^{8}$ La sensibilité à ces enjeux est d'autant plus grande que, outre l'environnement, c'est l'agriculture prise dans son ensemble, avec tout ce qu'elle représente y compris sur le plan de la conception de la société et des rapports à la nature, qui est en cause.
} 
d'OGM « contaminants » dans des lots de produits non OGM. On sent bien toute l'ambiguïté de ces normes qui se veulent générales et directives, alors que chaque cas de plante et de produit pose une question spécifique, dont il n'est pas certain que les normes fassent le traitement le plus juste intellectuellement, économiquement et socialement. Un long travail d'analyse, d'explication et $\mathrm{d}^{\prime}$ approfondissement reste donc à faire.

La traçabilité en matière d'OGM végétaux ne correspond à aucune exigence du point de vue de la biologie. Elle est purement et simplement un compromis de société destiné à gérer sur un plan sociologique et politique leur introduction dans chacune des sociétés concernées. À chacune de celles-ci d'en faire l'usage qu'elle souhaite et de se donner les moyens de le faire. Dans ce processus de caractère d'abord politique, les contributions que la biologie peut apporter sont d'ordre purement instrumental. Elles se situent à deux niveaux. Elle peut en premier lieu fournir des outils techniques (Tourte, 2001) pour la mise en œuvre et le suivi de la traçabilité : au niveau moléculaire (ADN), ce n'est ni plus difficile ni plus coûteux que ce que la justice utilise pour ses tests $\mathrm{ADN}$; cela aura par ailleurs les mêmes incertitudes. Elle peut en outre former les citoyens pour qu'ils apprennent à prendre la mesure de la traçabilité.

Au total, d'un point de vue biologique, le maintien de filières OGM et de filières non OGM, $y$ compris agrobiologiques, est parfaitement envisageable à deux conditions. Il suppose d'abord un investissement important sur le plan scientifique et technique; le prix en est élevé, mais on sait faire. Il suppose par ailleurs deux interventions politiques : d'une part, un contrôle strict de la circulation des produits; d'autre part, une gestion spatiale rigoureuse de la production. Cette dernière ne peut manquer de provoquer de multiples remaniements dans la répartition des cultures et des modes de culture, et ceci s'observe dans le monde entier (Crop Biotech Network, 2004).

\section{Conclusion}

Au total, si l'on se situe strictement sur le plan biologique, ce qui ressort des développements qui précèdent conduit à conclure :

- que les utilisations à des fins médicales ou agroalimentaires des OGM végétaux entraînent bien l'apparition de nouveaux risques, tant dans le domaine de la santé que dans celui de l'environnement,

- mais que, bien que nouveaux par nature, ces risques relèvent de connaissances et de savoir-faire - qu'il s'agisse de procédures d'acquisition de connaissances ou de contrôle du risque - connus, expérimentés et ayant fait leurs preuves, comme montré leurs limites, en dehors des OGM,
- qu'en conséquence, l'utilisation en vraie grandeur des OGM végétaux prolonge, plus qu'elle n'est en rupture avec elles, les évolutions antérieures dans les utilisations de la biologie, que ce soit en matière de santé ou d'agriculture,

- et qu'à condition d'être soigneusement réglementée et strictement encadrée sur le plan politique, non seulement elle ne représente en rien des risques supérieurs à ceux de ces dernières, mais elle est même au contraire susceptible d'en corriger les effets néfastes,

- que, bien évidemment, de nombreuses incertitudes persistent dans les connaissances concernant ces risques,

- mais qu'il en sera toujours ainsi compte tenu de l'extrême diversité et du renouvellement constant des cas de figure envisageables, tant du point de vue des OGM qui seront conçus et fabriqués, que des utilisations qui en seront faites et des conditions dans lesquelles ils seront utilisés,

- et que, de ce fait, la question n'est pas de savoir si les connaissances acquises en biologie et en ingénierie biologique, qui sont à la base des OGM végétaux, permettent ou non de se lancer d'ores et déjà dans l'utilisation en vraie grandeur des OGM végétaux,

- mais celle de se donner les moyens de mettre en œuvre, tant d'une manière générale que dans chaque cas particulier, les outils d'acquisition de connaissances et de contrôle du risque pour toute utilisation envisagée ou déjà mise en œuvre d'un OGM végétal, en application du principe de précaution,

- que, comme pour toute autre innovation, seule une utilisation aussi large que possible des OGM végétaux non seulement permettra d'en accroître la maîtrise, mais encore et surtout donnera à la diversité spatiale et temporelle des formes de leur intégration dans les systèmes de production d'une part, dans le processus d'évolution de la biodiversité d'autre part, l'occasion de s'exprimer; cela permettra de sortir du discours trop abstrait, parce que trop général, sur la question.

L'assurance du biologiste pourra surprendre, tant elle contraste avec les réticences, voire l'opposition résolue, que suscitent les OGM végétaux dans les opinions, chez les consommateurs et chez les responsables politiques, dans certains pays au moins. Le problème est de parvenir à distinguer, dans les raisons de ces réserves, celles qui renvoient au domaine de la biologie et celles qui ont trait aux évolutions et aux conceptions de la société, ainsi qu'aux représentations du vivant. C'est précisément l'objet de ce texte de tenter de faire le plus justement possible la part des choses.

Les OGM ont pris leur essor dans une période traversée par de multiples débats : question du mode de développement agricole susceptible de résoudre le problème de la pauvreté et de la faim dans les pays dits du Sud, question de la sûreté de l'alimentation dans les pays 
dits « développés », question de la biodiversité (de sa gestion, de sa protection et de son appropriation), question de la «mondialisation », question du rapport à la nature. Et, au bout de tout cela, remise en cause d'une certaine idée $\mathrm{du}$ « Progrès » et questions à propos du rôle de la science et de la responsabilité des scientifiques. Cela fait beaucoup.

Or, les OGM végétaux ont une histoire très liée à celle de grandes multinationales des secteurs de la santé et de l'agroalimentaire et à la course au profit à laquelle cellesci se livrent - avec ce que ceci suppose de précipitations et de tentatives de passage en force tant sur le plan de la mise au point des produits que de leur commercialisation (Kempf, 2003). Ils constituent par ailleurs une innovation technique apparemment radicale dans la manipulation du vivant, avec tout ce que cela signifie tant sur les plans philosophique et symbolique que du point de vue économique, social et, plus fondamentalement encore, sociétal. Ils se retrouvent donc, à travers l'un ou l'autre de leurs aspects, au cœur de cet ensemble de questions.

D'ailleurs, une bonne partie des débats a strictement trait aux incidences économiques et sociales de leurs utilisations sur les processus de développement et en particulier sur le développement agricole. Les arguments mis en avant sur ces différentes questions ont leurs fondements propres et n'impliquent en rien la biologie ; s'ils sont justiciables d'une référence scientifique, c'est aux sciences sociales que celle-ci renvoie. Mais des interférences ne peuvent manquer de se produire : faire apparaître les points faibles du dossier biologique peut être une façon de se donner des arguments pour contester le bien-fondé des OGM sur un plan strictement sociétal. Plus encore, le poids de la science dans l'idéologie dominante est tel qu'il faut aller jusqu'à invalider les arguments présentés en termes scientifiques pour pouvoir justifier de $s^{\prime} y$ opposer.

Or, ce que tendent à montrer les développements qui précèdent, c'est que cette vision des choses est réductrice et ne permet donc pas de poser les bonnes questions. Ce n'est pas seulement une entité technique et scientifique (à savoir les plantes OGM) qui est en cause, mais toute une démarche fondée sur une dynamique en boucles entre la recherche et les évolutions de la société. On aura noté que la plus vive des questions soulevées (celle des conséquences écologiques de la culture d'OGM végétaux en plein champ) est renvoyée dans le champ du social par le biologiste : c'est là toute une représentation du vivant qui est en cause et il est clair que celle du biologiste est aux antipodes de celle qui prévaut même dans la plupart des sociétés dites modernes. Les discours qui récusent le principe même de l'utilisation des OGM - que nous n'avons pas examinés ici - illustrent encore plus fortement cette rupture de continuité entre ces deux représentations du vivant. On aura également noté qu'une des conditions de la maîtrise des OGM végétaux, telle qu'elle est socialement souhaitée, est leur encadrement strict tant sur un plan réglementaire que politique (tant au niveau local qu'international). Or, on peut douter que le fonctionnement des sociétés puisse réaliser ces conditions. Il suffit d'observer la façon dont la question des OGM est actuellement traitée au niveau international pour le comprendre et s'en convaincre (la question du changement climatique est tout aussi instructive).

Dès lors, c'est tout le modèle fondé sur la construction d'une entité biologique pure, abstraction faite des conditions sociales de son utilisation et de sa circulation, qui est remis en cause. C'est l'entité biologique inscrite dans les règles qui gouvernent les systèmes sociaux dans lesquels elle opère (c'est-à-dire dans lesquels à la fois elle se réalise et elle apporte sa contribution au processus social la justifiant) qui doit être l'objet réel de l'analyse.

Dans une telle perspective, les incertitudes scientifiques sont prises en compte puisqu'elles conditionnent le devenir social de l'entité biologique en cause, mais elles ne sont pas simplement vues comme des problèmes scientifiques ou techniques à résoudre. Elles sont vues aussi comme les fondements d'enjeux de société qu'il revient alors aux sciences sociales de mettre en évidence pour qu'ils fassent partie à part entière d'une analyse qui restitue la dualité intrinsèque de l'objet : en effet, celui-ci n'est-il pas biologiquement ce qu'il est parce qu'il est une construction sociale et, dans le même temps, socialement (lato sensu) appréhendé comme il l'est parce que construction biologique? C'est de cette dualité insécable que «parlent» les débats. C'est cette unité à la fois duelle et indissoluble qu'ils proclament et revendiquent. En l'ignorant, c'est-à-dire en fait en la niant, la recherche, non seulement ne contribuerait pas à la clarté du débat, mais le rendrait encore plus confus en y introduisant l'artefact des limites qu'elle subit dans ses démarches $\mathrm{du}$ fait de la division du travail sur la base de laquelle elle produit les connaissances (Friedberg, 2002). Reconnaître cette dualité, l'assumer et se donner les moyens de l'analyser est indispensable pour parvenir à une compréhension non seulement de la question des OGM prise dans son ensemble, mais de celle, générale et qui revient actuellement de plus en plus fréquemment, de la place et du rôle de la science quand l'évolution des connaissances débouche sur des innovations techniques remettant en cause des rapports de production, des systèmes de valeurs, des pratiques et jusqu'aux fondements symboliques mêmes de la société.

\section{Références}

Académie des sciences, 2002. Les Plantes génétiquement modifiées, RST n ${ }^{\circ} 13$, Paris, Tec \& Doc.

Académie des sciences, 2003. De la transgenèse animale à la biothérapie chez l'homme, RST no 14 , Paris, Tec \& Doc. 
Bizet, J., Pastor, J.-M., 2003. Quelle politique des biotechnologies pour la France?, Les Rapports du Sénat, 301.

Boyaval, J., 2003. Organismes génétiquement modifiés : une réglementation renforcée, Paris, BIMA, décembre, 24-26.

Commissariat général au plan, rapport du groupe présidé par B. Chevassus-au-Louis, 2001. OGM et agriculture : options pour l'action publique, Paris, La Documentation française.

Crop Biotech Network, 2004. Global status of commercialized transgenic crops : 2003, Crop Biotech Update Special Edition, http://www.isaa.org/kc

Friedberg, C., 2002, Pour un savoir intégré : quelques réflexions à propos du débat public sur les OGM en France, Natures Sciences Sociétés, 10, 1, 54-58.

INRA, 2002. OGM et environnement, séminaire de restitution de l'AIP "OGM et environnement », Paris, INRA Éditions.

Joly, P.-B., Assouline, G., Kreziak, D., Lenarié, J., Marris, C., Roy, A., 2000. L'Innovation controversée : le débat public sur les OGM en France. Rapport du Cride, Paris, INRA Éditions.
Joly, P.-B., Hervieu, B., 2003. La marchandisation du vivant, Futuribles, 292, 5-29.

Kempf, H., 2003. La Guerre secrète des OGM, Paris, Le Seuil.

Ministère de l'Écologie et du Développement durable, 2003. Les Organismes Génétiquement Modifiés (OGM). Les fiches : http://www.environnement.gouv.fr

Mounolou, J.-C., Fridlansky, F., 2002. OGM : attentes inquiètes, attentes tranquilles. Un tour d'horizon de biologistes, Natures Sciences Sociétés, 10, 1, 46-53.

Semences et biodiversité, 2003. Colloque du Groupement interprofessionnel des semences et des plants, http://www.gnis.fr

Ternois, N., 2004. Coexistence entre colza OGM et colza non OGM possible mais contraignante, Cultivar, 566, 24.

Tourte, Y., 2001. Les OGM : la transgenèse chez les plantes, Paris, Dunod. 